\title{
Gapless hinge states from adiabatic pumping of axion coupling
}

Olsen, Thomas; Rauch, Tomáš; Vanderbilt, David; Souza, Ivo

Published in:

Physical Review B

Link to article, DOI:

10.1103/PhysRevB.102.035166

Publication date:

2020

Document Version

Publisher's PDF, also known as Version of record

Link back to DTU Orbit

Citation (APA):

Olsen, T., Rauch, T., Vanderbilt, D., \& Souza, I. (2020). Gapless hinge states from adiabatic pumping of axion coupling. Physical Review B, 102(3), [035166]. https://doi.org/10.1103/PhysRevB.102.035166

\section{General rights}

Copyright and moral rights for the publications made accessible in the public portal are retained by the authors and/or other copyright owners and it is a condition of accessing publications that users recognise and abide by the legal requirements associated with these rights.

- Users may download and print one copy of any publication from the public portal for the purpose of private study or research.

- You may not further distribute the material or use it for any profit-making activity or commercial gain

- You may freely distribute the URL identifying the publication in the public portal

If you believe that this document breaches copyright please contact us providing details, and we will remove access to the work immediately and investigate your claim. 


\title{
Gapless hinge states from adiabatic pumping of axion coupling
}

\author{
Thomas Olsen, ${ }^{1, *}$ Tomáš Rauch, ${ }^{2,3}$ David Vanderbilt $\odot,{ }^{4}$ and Ivo Souza $\oplus^{3,5}$ \\ ${ }^{1}$ Computational Atomic-Scale Materials Design, Department of Physics, Technical University of Denmark, 2800 Kgs. Lyngby, Denmark \\ ${ }^{2}$ Friedrich-Schiller-University Jena, 07743 Jena, Germany \\ ${ }^{3}$ Centro de Física de Materiales, Universidad del País Vasco, 20018 San Sebastián, Spain \\ ${ }^{4}$ Department of Physics and Astronomy, Rutgers University, Piscataway, New Jersey 08854, USA \\ ${ }^{5}$ Ikerbasque Foundation, 48013 Bilbao, Spain
}

(Received 16 June 2020; accepted 13 July 2020; published 31 July 2020)

\begin{abstract}
We demonstrate that chiral hinge modes naturally emerge in insulating crystals undergoing a slow cyclic evolution that changes the Chern-Simons axion angle $\theta$ by $2 \pi$. This happens when the surface (not just the bulk) returns to its initial state at the end of the cycle, in which case it must pass through a metallic state to dispose of the excess quantum of surface anomalous Hall conductivity pumped from the bulk. If two adjacent surfaces become metallic at different points along the cycle, there is an interval in which they are in topologically distinct insulating states, with chiral modes propagating along the connecting hinge. We illustrate these ideas for a tight-binding model consisting of coupled layers of the Haldane model with alternating parameters. The surface topology is determined in a slab geometry using two different markers, surface anomalous Hall conductivity and surface-localized charge pumping (flow of surface-localized Wannier bands), and we find that both correctly predict the appearance of gapless hinge modes in a rod geometry. When viewing the axion pump as a fourdimensional crystal with one synthetic dimension, the hinge modes trace Fermi arcs in the Brillouin zone of the two-dimensional hinge connecting a pair of three-dimensional surfaces of the four-dimensional crystal.
\end{abstract}

DOI: 10.1103/PhysRevB.102.035166

\section{INTRODUCTION}

The electronic states of crystalline insulators can be characterized by certain geometric properties of the wave functions that have measurable consequences [1]. For example, in one-dimensional (1D) insulators the manifold of valence states carries a Berry phase [2]:

$$
\gamma=\int_{0}^{2 \pi} \operatorname{Tr}\left[A^{k}\right] d k
$$

where $k$ between zero and $2 \pi$ is the reduced wave vector in the Brillouin zone (BZ), and the integrand is the trace of the Berry connection matrix $A_{m n}^{k}=i\left\langle u_{k m} \mid \partial_{k} u_{k n}\right\rangle$ over the valence bands. Although $\operatorname{Tr}\left[A^{k}\right]$ is not invariant under gauge transformations among the valence states, its integral $\gamma$ is invariant modulo $2 \pi$. Physically, $\gamma$ describes the electronic contribution to the electric polarization as

$$
P=-e \frac{\gamma}{2 \pi}
$$

where $e>0$. Accordingly, the bulk polarization is itself only defined modulo $e$ in one dimension $[3,4]$.

The quantum of indeterminacy present in Eq. (1) allows for the possibility of changing $\gamma$ gradually by a multiple of $2 \pi$ during a slow cyclic evolution, resulting in the transport of an integer number $C_{1}$ of electrons over one lattice constant [5]. If the Hamiltonian is parametrized by an angle $\phi$, the total

*tolsen@fysik.dtu.dk change in $\gamma$ over one cycle from $\phi=0$ to $2 \pi$ is given by

$$
\Delta \gamma=\int_{0}^{2 \pi} d \phi \int_{0}^{2 \pi} d k \operatorname{Tr}\left[\Omega^{\phi k}\right]=2 \pi C_{1} .
$$

$C_{1}$ is known as the first Chern number, and when it is nonzero the cycle is called a Thouless pump. In Eq. (3), $\Omega_{m n}^{\phi k}=\partial_{\phi} A_{m n}^{k}-\partial_{k} A_{m n}^{\phi}-i\left[A^{\phi}, A^{k}\right]_{m n}$ is the covariant Berry curvature matrix of the valence bands in $(\phi, k)$ space. When this $(\phi, k)$ space is viewed as an effective two-dimensional (2D) momentum space, $2 \pi C_{1}$ becomes a quantized Berry flux through the corresponding $2 \mathrm{D} \mathrm{BZ}$.

The Chern number $C_{1}$ can be defined in exactly the same way for real 2D insulators, and those for which it is nonzero are known as (first) Chern insulators, or quantum anomalous Hall insulators. Chern insulators were introduced by Haldane using a tight-binding model [6], and have been realized experimentally in magnetically doped thin films $[7,8]$. They are characterized by a quantized anomalous Hall conductivity (AHC) of $\left(e^{2} / h\right) C_{1}$, and by the presence of $\left|C_{1}\right|$ chiral edge modes crossing the bulk gap.

In three dimensions, the valence bands of insulating crystals carry another global geometric property. It is known as the Chern-Simons axion angle $\theta$, and can be expressed as $[9,10]$

$$
\theta=-\frac{1}{4 \pi} \int_{\mathrm{BZ}} \varepsilon_{a b c} \operatorname{Tr}\left[A^{a} \partial_{b} A^{c}-i \frac{2}{3} A^{a} A^{b} A^{c}\right] d^{3} k,
$$

where each $k_{a}$ runs between zero and $2 \pi, \partial_{a}=\partial / \partial k_{a}$, and $A_{m n}^{a}$ is the corresponding Berry connection along lattice direction $a$. The axion angle describes an isotropic contribution $\alpha_{\alpha \beta}^{\text {iso }}=\left(e^{2} / h\right)(\theta / 2 \pi) \delta_{\alpha \beta}$ to the linear 
(a)

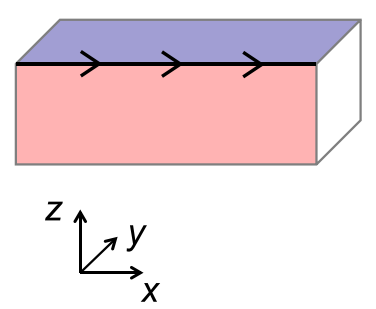

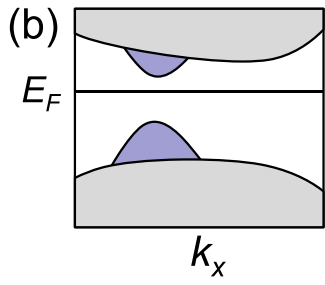

(e)

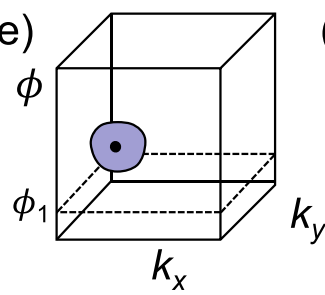

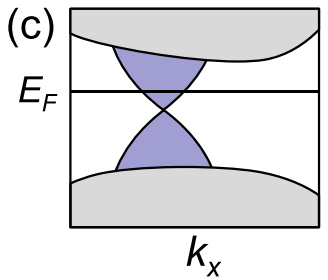

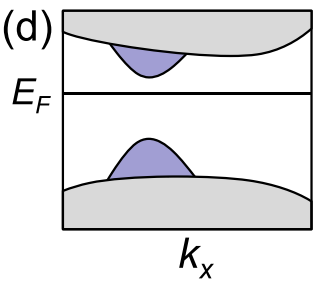

(f)
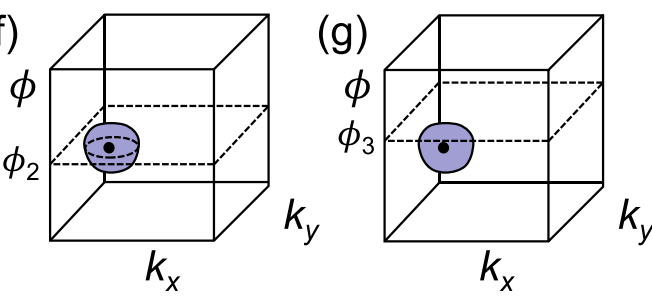

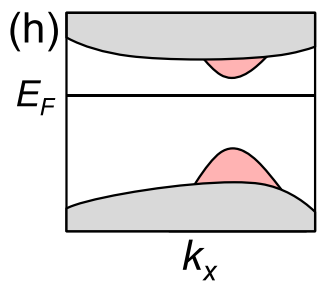

(i)

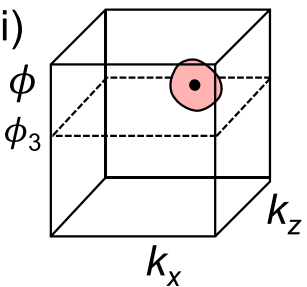

FIG. 1. (a) Real-space view of a sample of an insulating material undergoing an axion pumping cycle parametrized by $\phi$. The sample is obtained by terminating a bulk crystal at two semi-infinite surfaces oriented normal to $+\hat{\mathbf{z}}$ and $-\hat{\mathbf{y}}$ (blue and red shadings, respectively), meeting at an $x$-directed hinge that may harbor chiral modes (arrows). (b)-(d) Hinge-projected band structure focusing on surface states on the $+\hat{\mathbf{z}}$-oriented surface (blue shading), for three increasing values of $\phi$. Gray shading represents projected bulk states. (e)-(g) View in $\left(k_{x}, k_{y}, \phi\right)$ space, for the choice of Fermi level indicated in (b)-(d); blue indicates the "Fermi surface" in $\left(k_{x}, k_{y}, \phi\right)$ space enclosing the electron pocket; the solid dot is the Weyl point, corresponding to the nodal touching of surface valence and conduction bands at the critical parameter value $\phi_{2}$. The dashed lines in (e)-(g) indicate the $\phi$ values used in (b)-(d), respectively. (h, i) Same as (d) and (g), but focusing on states localized on the surface normal to $-\hat{\mathbf{y}}$.

magnetoelectric coupling $\alpha_{\alpha \beta}=\partial P_{\alpha} / \partial B_{\beta}=\partial M_{\beta} / \partial \mathcal{E}_{\alpha}$. Like the Berry phase, the axion angle is gauge invariant only modulo $2 \pi$ and can change gradually by multiples of $2 \pi$ during slow cyclic evolutions. The net change in $\theta$ over one cycle parametrized by $\phi \in[0,2 \pi]$ is given by

$$
\Delta \theta=\frac{1}{16 \pi} \int_{\mathrm{BZ}} d^{3} k \int_{0}^{2 \pi} d \phi \varepsilon_{a b c d} \operatorname{Tr}\left[\Omega^{a b} \Omega^{c d}\right]=2 \pi C_{2},
$$

where the indices run over $k_{x}, k_{y}, k_{z}, \phi$. The integer $C_{2}$ is called the second Chern number, and when it is nonzero the cycle is referred to as an axion pump [11]. Like $2 \pi C_{1}$ given by Eq. (3), $2 \pi C_{2}$ can be viewed as a quantized Berry flux through an effective BZ, which is now that of a parent four-dimensional (4D) insulator.

In this paper, we ask what general features one can expect to see in the boundary spectrum during an axion pumping cycle when both the bulk and the surface return to their initial states at the end of the cycle. The evolution of the surface spectrum under these circumstances was studied previously [12]. In the present paper we turn our attention to the hinge band structure, that is, the spectrum of 1D modes localized at the boundaries between contiguous surface facets.

We find that chiral hinge states appear generically in the course of cyclic evolutions characterized by a nonzero $C_{2}$ invariant. In contrast to the hinge states in intrinsic higher-order topological insulators $[13,14]$, their occurrence does not rely on the presence of certain bulk crystallographic symmetries, but only on the global Chern topology of the pumping cycle.

The paper is organized as follows. In Sec. II we provide a qualitative discussion of the main ideas. We then illustrate them for a concrete tight-binding model in Sec. III, where we use various tools to predict from slab calculations the occurrence of gapless hinge modes, and to illuminate the concept of surface topology. We conclude in Sec. IV with a summary and outlook.

\section{QUALITATIVE DISCUSSION}

In this section we examine the generic behavior of boundary states during an axion pumping cycle, making no special assumptions about the presence of symmetries or the position of the Fermi level $E_{\mathrm{F}}$ in the gap, and ask what general features one can expect to see in the surface and hinge band structures during an axion pump evolution cycle.

We consider for simplicity an orthorhombic structure with primitive lattice vectors along the Cartesian axes, and work with reduced wave vectors $\left(k_{x}, k_{y}, k_{z}\right)$ with each $k_{j}$ between zero and $2 \pi$. To these we can add the adiabatic Hamiltonian parameter $\phi$ whose evolution from zero to $2 \pi$ controls the axion pump, so that we can also think in terms of a 4D insulator with a second Chern number $C_{2}$ in $\left(k_{x}, k_{y}, k_{z}, \phi\right)$ momentum space. In the present discussion we shall assume $C_{2}=1$, so that one quantum of axion coupling is pumped during the adiabatic cycle.

\section{A. Surface states}

Consider the system shown in Fig. 1(a) —a crystal terminated at two semi-infinite surfaces normal to $+\hat{\mathbf{z}}$ and $-\hat{\mathbf{y}}$, meeting at an $x$-directed hinge. In preparation for the discussion of hinge-localized states in the next subsection, here we consider the band structures of the two surfaces, but projected as though seen from the hinge.

We focus first on the top surface (unit normal $+\hat{\mathbf{z}}$ ). The evolution of its hinge-projected band structure is sketched in panels (b)-(d) for three increasing values of $\phi$ in the region where the metallization occurs. These are labeled as $\phi_{1}, \phi_{2}$, and $\phi_{3}$, corresponding to panels (e)-(g), respectively, where the locus of electron-occupied surface states is indicated in the three-dimensional (3D) $\left(k_{x}, k_{y}, \phi\right)$ space. As a reminder, the surface is required to become metallic over some range of $\phi$. 
This follows because we assume that the surface Hamiltonian (as well as the bulk one) returns to itself at the end of the $\phi$ loop, so that the quantum of AHC that is pumped to this surface has to be removed by a metallic interval [12]. Typically this happens as shown in panels (b)-(d). That is, surface states penetrate into the gap with increasing $\phi$, leading to the formation of a nodal touching in $\left(k_{x}, k_{y}\right)$ space at the critical parameter value $\phi_{2}$, after which the gap reopens to restore an insulating surface. When viewed in $\left(k_{x}, k_{y}, \phi\right)$ space, that nodal touching becomes a Weyl point, indicated by the solid dot in panels (e)-(g).

If one would follow the evolution of the surface AHC by computing the contributions only up to the nodal point, one would observe a sudden jump by $e^{2} / h$ when passing through $\phi_{2}$. This jump is precisely by the amount needed to return the surface AHC to its initial value at the end of the cycle. With the indicated Fermi-level position, however, the change occurs continuously. An electron pocket first appears when the conduction band minimum drops below $E_{\mathrm{F}}$, somewhere between panels (b) and (c); it grows, then shrinks and disappears somewhere between (c) and (d). This behavior is visualized in $3 \mathrm{D}\left(k_{x}, k_{y}, \phi\right)$ space in panels (e)-(g), with the dashed rectangles showing the $\phi$ values corresponding to panels (b)-(d), respectively.

At any value of $\phi$ for which the surface electron pocket exists, such as that shown by the horizontal cut in Fig. 1(f), the contribution of that pocket to the surface $\mathrm{AHC}$ is proportional to the Berry phase computed around its boundary. This phase evolves by $2 \pi C_{1}^{\mathrm{FS}}$ from the creation to the destruction of the pocket, where $C_{1}^{\mathrm{FS}}$ is the first Chern number (1, in our case) on the spheroidal Fermi surface shown in panels (e)-(g) of Fig. 1.

We can regard any one of those three panels as showing the Fermi-surface structure of the 3D $\left(k_{x}, k_{y}, \phi\right)$ system corresponding to the $z$-terminated 3D surface of a $4 \mathrm{D}\left(k_{x}, k_{y}, k_{z}, \phi\right)$ second-Chern insulator. We see a Fermi pocket with nonzero first Chern index surrounding a Weyl node, shown as a dark central point in each panel. This looks very much like a picture of a Weyl semimetal [15], but with one crucial difference. In a true 3D system, the Nielsen-Ninomiya theorem [16] requires that the chiralities of the Weyl nodes must sum to zero over the $3 \mathrm{D} \mathrm{BZ}$. The violation we see here is an example of an anomaly; since we are at the surface of a topological $4 \mathrm{D}$ insulator, the reasoning used to prove the Nielsen-Ninomiya theorem no longer applies. In fact, the sum of chiralities is necessarily equal to the 4D bulk second Chern number, that is, $C_{1}^{\mathrm{FS}}=C_{2}$. Hence, every $3 \mathrm{D}$ surface facet of a $4 \mathrm{D}$ second-Chern insulator must show the same excess of chirality $[9,12]$. In particular, every 3D surface must be metallic, in analogy to the $1 \mathrm{D}$ surfaces of a $2 \mathrm{D}$ first-Chern insulator.

Panels (h) and (i) of Fig. 1 show similar plots at the same $\phi=\phi_{3}$ value as in panels (d) and (g), but now for the surface with unit normal $-\hat{\mathbf{y}}$. Here we assume that the metallic interval has not yet begun, so the electron pocket corresponding to this surface lies above the $\phi=\phi_{3}$ plane. Incidentally, if the Fermi-level position had been chosen lower in the gap, the metallic interval of $\phi$ could correspond to the temporary creation of a hole pocket instead, on either or both of the surface facets; entirely parallel arguments apply in these cases.
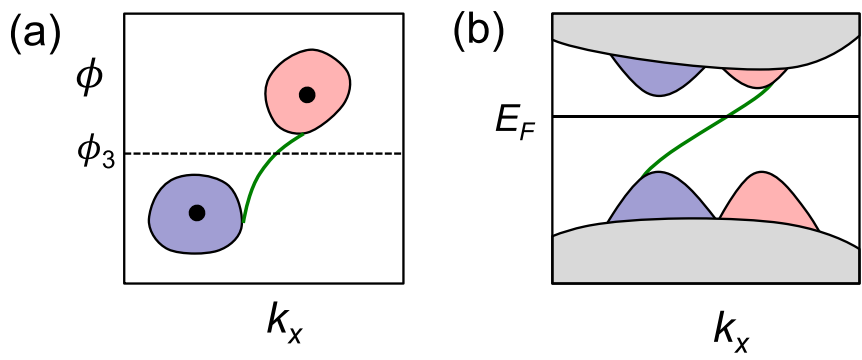

FIG. 2. (a) Hinge-projected Fermi surface of the system depicted in Fig. 1(a), plotted in $\left(k_{x}, \phi\right)$ space. Blue and red electron pockets correspond to surface states on the $+\hat{\mathbf{z}}$ - and $-\hat{\mathbf{y}}$-oriented surfaces, as shown in Figs. 1(g) and 1(i), respectively. The Fermi-arc state is shown in green. (b) Hinge-projected band-structure plot vs $k_{x}$ [at $\phi=\phi_{3}$, indicated by the dashed line in (a)]. The gray, blue, and red regions are the projected bulk, $+\hat{\mathbf{z}}$-surface, and $-\hat{\mathbf{y}}$-surface states shown in Figs. 1(d) and 1(f), and the green line is the chiral hinge state.

\section{B. Hinge states}

Now consider the $x$-directed hinge adjoining the $y$ - and $z$ oriented surface facets discussed above (we refer to it as a " $y \mid z$ hinge"). Figure 2(a) shows the locus of points on the $\left(k_{x}, \phi\right)$ plane where there are states at $E_{\mathrm{F}}$, that is, the hinge-projected Fermi surface plotted as a function of $\phi$. The blue region is the projection onto the $\left(k_{x}, \phi\right)$ plane of the Fermi surface in $\left(k_{x}, k_{y}, \phi\right)$ space of the $+\hat{\mathbf{z}}$-oriented surface [Fig. 1(g)]. Likewise, the red region is the projection of the $\left(k_{x}, k_{z}, \phi\right)$ Fermi surface of the $-\hat{\mathbf{y}}$-oriented surface [Fig. 1(i)] adjoining the $+\hat{\mathbf{z}}$ surface at the hinge. Each electron pocket encloses a Weyl point, and these bring opposite chiral charges due to the fact that positive circulations on the $+\hat{\mathbf{z}}$ - and $-\hat{\mathbf{y}}$-oriented surfaces correspond to positive and negative transport, respectively, at the hinge.

The Nielsen-Ninomiya theorem is now satisfied, since the total chirality of all Weyl points projecting onto the $\left(k_{x}, \phi\right)$ plane necessarily vanishes. However, the separation of chiral charges between the two pockets requires the presence of a Fermi arc connecting them, as shown by the green line in Fig. 2(a), just as for the case of a Fermi arc at the surface of a Weyl semimetal [15]. Indeed, the count of Fermi arcs and geometry of attachment must follow the same rules outlined by Haldane [17]. The full hinge-projected band structure at $\phi=\phi_{3}$ is illustrated in Fig. 2(b), showing the hinge-localized state that crosses from the valence to the conduction manifold and gives rise to the Fermi arc.

Of course, at other points along the adiabatic pumping cycle, corresponding to different values of $\phi$, the chiral hinge mode may be absent, as above the red region or below the blue region in Fig. 2(a). At some other $\phi$ values, the presence of hinge states will be obscured by degeneracy with the continuum of surface states. In some cases these regions of metallic surface behavior could be much more extensive than as sketched above, hiding the hinge electronic structure almost completely. However, we can be sure of the existence of at least one Weyl-node-surrounding hole or electron pocket arising from each adjoined surface, and a required Fermi arc state connecting them somewhere in the $\left(k_{x}, \phi\right)$ space (unless 
the two pockets overlap when projected into this space). In this sense, the presence of Fermi arcs and chiral hinge channels at some stage of a second-Chern pumping cycle is generic. Nevertheless, in some model systems the surface nodal touchings may occur at the same $\phi$ on different surfaces, especially if the critical $\phi$ is pinned to a specific value at which the system has some higher symmetry, such as time reversal or mirror symmetry. However, in the context of the dramatic surface-facet variations in structural and chemical properties that are typical of real materials, such commonality of the critical $\phi$ values appears unlikely.

The observations presented above serve to illustrate the very close analogy between the physics on the $2 \mathrm{D}$ "hinge" of a $4 \mathrm{D}$ second-Chern topological insulator, and at the 2D surface of a 3D Weyl semimetal.

\section{NUMERICAL STUDY OF A TOY MODEL}

\section{A. The alternating Haldane model}

To illustrate the physics described above, we study the tight-binding model for an axion pump introduced in Ref. [12], consisting of alternating layers of the Haldane model [6]. The on-site energies are modulated by an angle $\phi$ in such a way that for $-\pi / 2<\phi<\pi / 2$ the first Chern numbers vanish on all layers, while for $\pi / 2<\phi<3 \pi / 2$ they alternate between +1 and -1 , for isolated layers. To prevent the layers from becoming metallic at $\phi=\pi / 2$ and $3 \pi / 2, \phi$-dependent interlayer couplings are introduced. As $\phi$ goes from zero to $2 \pi$, the system is carried along a gapped circuit that encloses a gapless point in parameter space, and the axion angle $\theta$ increases gradually from zero to $2 \pi$ [12].

When viewed along the stacking direction $z$, the model consists of coupled chains that project onto the honeycomb sites on each layer, with alternating on-site energies and hoppings along $z$. In the limit of vanishing interchain coupling, the chain Hamiltonian is identical to the Rice-Mele model of alternating site energies and hopping strengths [18], which realizes a Thouless pump [4]. Chains passing through the $A$ and $B$ sites have equal and opposite first Chern numbers in $\left(\phi, k_{z}\right)$ space, so that no net charge is transported along $z$ over one cycle. Depending on the choice of parameters, the magnitude of those Chern numbers is either zero or one.

The 2D unit cell of each layer is spanned by the lattice vectors $\mathbf{a}_{1}=a \hat{\mathbf{x}}$ and $\mathbf{a}_{2}=a \hat{\mathbf{x}} / 2+\sqrt{3} a \hat{\mathbf{y}} / 2$, with orbitals sitting on the honeycomb sites $\mathbf{t}_{\mathrm{A}}=\mathbf{a}_{1} / 3+\mathbf{a}_{2} / 3$ and $\mathbf{t}_{\mathrm{B}}=2 \mathbf{a}_{1} / 3+$ $2 \mathbf{a}_{2} / 3$. The Hamiltonian for an isolated layer indexed by $p$ is

$$
\begin{aligned}
H_{p}= & (-1)^{p} \Delta \sum_{i} \gamma_{i} c_{p i}^{\dagger} c_{p i}+t \sum_{\langle i j\rangle} c_{p i}^{\dagger} c_{p j} \\
& +(-1)^{p} \sum_{\langle i j\rangle} i v_{i j} c_{p i}^{\dagger} c_{p j},
\end{aligned}
$$

where $i$ and $j$ label the sites, with $\gamma_{i}= \pm 1$ if site $i$ belongs to the A or B sublattice. $\langle i j\rangle$ and $\langle i j\rangle\rangle$ denote pairs of first and second nearest-neighbor sites, with each pair appearing twice. The first and second terms contain the on-site energies and nearest-neighbor hoppings, respectively, and the third describes a pattern of staggered magnetic fluxes generated by complex second-neighbor hoppings of unit magnitude. Therein, $v_{i j}=+1(-1)$ if the hopping direction from $j$ to $i$ is right handed (left handed) around the center of a plaquette. The $(-1)^{p}$ factor in the first term reverses the energies of sites on the same sublattice in adjacent layers, while the same factor in the third term reverses the pattern of magnetic fluxes, and with it the first Chern numbers on consecutive layers. The hopping magnitude in the third term has been set to unity as a reference, and each 2D layer undergoes a Chern transition between topological and trivial phases at $\Delta= \pm 3 \sqrt{3}$. At $\Delta=$ $3 \sqrt{3}$ the gap-closing transition occurs at the high-symmetry point $\bar{K}=(4 \pi / 3 a) \hat{\mathbf{x}}$ in the $2 \mathrm{D} \mathrm{BZ}$, and at $\Delta=-3 \sqrt{3}$ it occurs at $\overline{K^{\prime}}=-(4 \pi / 3 a) \hat{\mathbf{x}}$.

The full 3D model has $\mathbf{a}_{3}=c \hat{\mathbf{z}}$ as the third lattice vector, and two layers per unit cell. The layers $p=0,1$ are located at $z=-c / 4, c / 4$, and the Hamiltonian reads

$$
H_{\text {bulk }}=\sum_{p}\left\{H_{p}+\left[1+(-)^{p} t^{\prime}\right] \sum_{i} \gamma_{i}\left(c_{p i}^{\dagger} c_{p+1, i}+\text { H.c. }\right)\right\} \text {, }
$$

where the second term describes the interlayer (intrachain) coupling and "H.c." stands for "Hermitian conjugate." We choose $t=-4.0$, and parametrize $\Delta$ and $t^{\prime}$ according to

$$
\begin{aligned}
\Delta & =3 \sqrt{3}+2 \cos \phi, \\
t^{\prime} & =0.4 \sin \phi .
\end{aligned}
$$

The presence of a nonzero $t^{\prime}$ introduces an alternation of interlayer hopping strengths that keeps the system gapped as $\phi$ passes through $\pi / 2$ and $3 \pi / 2$ where the topological transitions occur in the isolated layers. The bulk spectrum is therefore gapped everywhere along the adiabatic cycle parametrized by $\phi$, encircling a gapless point at $\left(\Delta, t^{\prime}\right)=$ $(3 \sqrt{3}, 0)$.

The model has neither time reversal (TR) nor inversion symmetry at generic $\phi$. However, the Hamiltonian is invariant under an antiunitary operator

$$
\Lambda=K i \tau_{y} \sigma_{x}=K \tau_{z} I
$$

where $K$ is complex conjugation and $\tau_{j}$ and $\sigma_{j}$ are the $j$ th Pauli matrices acting in the layer and sublattice spaces, respectively. ${ }^{1}$ Inversion about a hexagonally centered point midway between the layers is represented by $I=\tau_{x} \sigma_{x}$, so the second equality expresses $\Lambda$ as $I$ followed first by a sign reversal of all amplitudes on odd layers, then by scalar TR. Because $\Lambda$ is antiunitary and squares to -1 , it acts the way inversion times TR does in a spinor system, forcing the four energy bands to come in two Kramers-degenerate pairs. At the points $\phi=0$ and $\pi$ where the alternation $t^{\prime}$ of the interlayer hoppings vanishes, the model acquires two additional symmetries: mirror symmetry $M_{z}(z \rightarrow-z)$ about the layers, and time-reversal $K$ combined with a twofold rotation $C_{2}^{y}$ about an axis lying on the atomic layers and pointing along the armchair edges.

The evolution with $\phi$ of the axion angle is shown in Fig. 3. $\theta$ increases gradually from zero to $2 \pi$ over one cycle, corresponding to $C_{2}=1$ in Eq. (5). Both $M_{z}$ and $K C_{2}^{y}$ take $\theta$ into $-\theta$, constraining $\theta$ to be zero $\bmod \pi$ at $\phi=0$ and

\footnotetext{
${ }^{1}$ We thank N. Varnava for pointing out this symmetry of the model.
} 


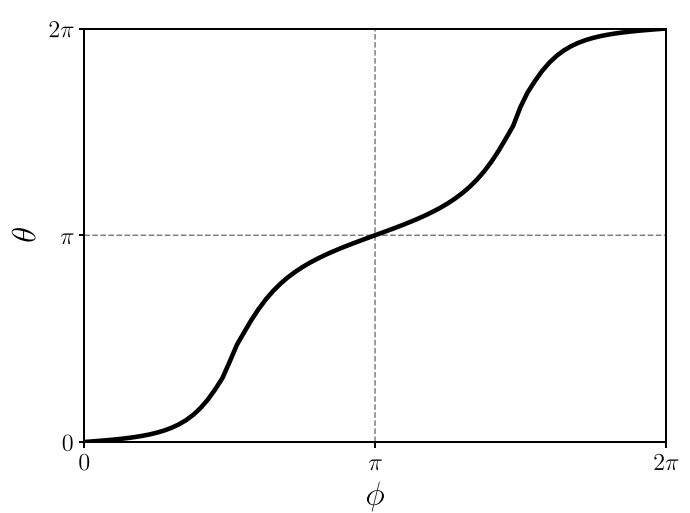

FIG. 3. Pumping of the axion angle $\theta$ by $2 \pi$ in the alternating Haldane model.

$\pi$, consistent with the figure. At $\phi=0$ the system is a topologically trivial insulator with $\theta=0$. Instead, at $\phi=\pi$ it is a topological crystalline insulator with $\theta=\pi$ (a "generalized axion insulator" in the sense of Ref. [19]), harboring metallic states on surfaces that preserve either $M_{z}$ or $K C_{2}^{y}$ symmetry, or both $[12,19]$.

\section{B. Surface topological transitions and surface anomalous Hall conductivity}

We study three types of slabs, shown schematically in Fig. 4. The ones in the left and middle panels are terminated along $z$, and the one on the right is terminated along $y$. In the left panel the stacking unit is a cell with boundaries at $z=$ $\pm 1 / 2$ (in units of $c$ ), enclosing layers located at $z= \pm 1 / 4$. With this " $z_{1 / 2}$ termination," the Chern numbers of the top and bottom layers (when isolated) are $C_{1}= \pm 1$, respectively, in the interval $\pi / 2<\phi<3 \pi / 2$. In the middle panel the stacking unit is a cell with boundaries at $z=0$ and 1 and enclosing layers at $z=1 / 4$ and $3 / 4$. With this " $z_{0}$ termination," the top and bottom layers have Chern numbers $C_{1}=\mp 1$, respectively, in the same interval. Finally, in the right panel we have a slab with a " $y_{0}$ termination" consisting of zigzag edges on every layer.

For each type of slab we perform the cyclic evolution described by Eq. (8), with the surfaces returning to their initial states along with the bulk. The surfaces must then pass through metallic states to dispose of the quantum of surface
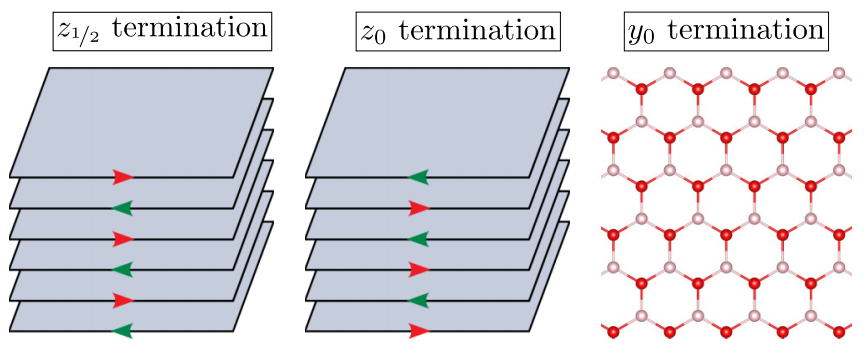

FIG. 4. Left and middle: Inequivalent $z$-terminated slabs of the alternating Haldane model. Arrows indicate edge-mode chiralities on the uncoupled layers for $\pi / 2<\phi<3 \pi / 2$. Right: One layer of a $y_{0}$-terminated slab, with zigzag edges (top view).
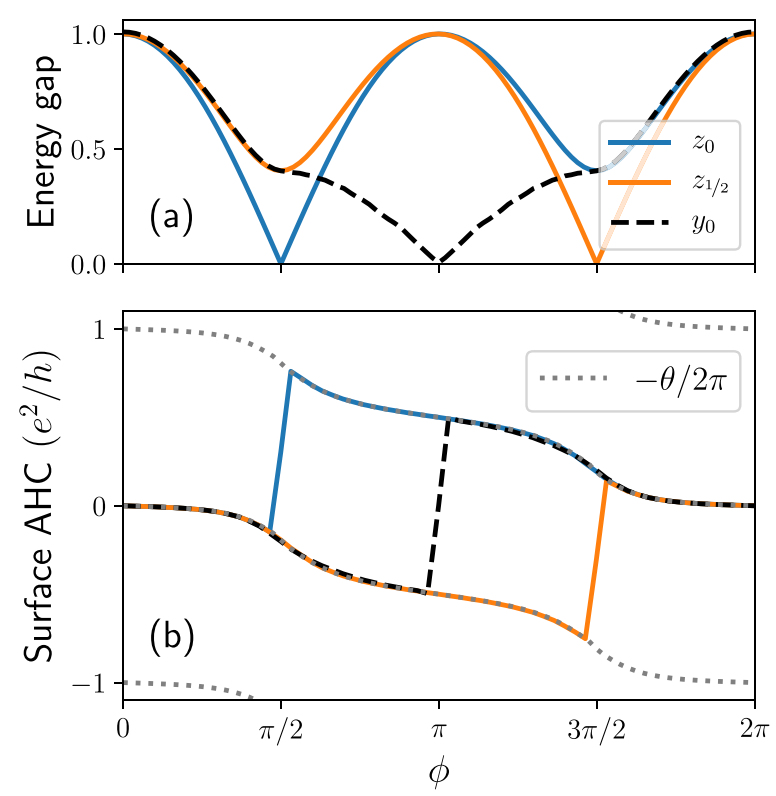

FIG. 5. (a) Evolution of the minimum energy gap vs $\phi$ in slabs of the alternating Haldane model, for the three types of surface terminations pictured in Fig. 4. The gap closures occur at the surfaces. (b) Evolution of the surface AHC of each slab. Dotted lines are different branches of the bulk axion coupling, plotted as $-\theta / 2 \pi$ according to Eq. (10). The finite slopes of the discrete jumps at $\phi=\pi / 2, \pi$, and $3 \pi / 2$ are artifacts of the finite step size used for $\phi$ in the calculation.

AHC pumped from the bulk. To visualize the gap closure, we plot in Fig. 5(a) the minimum energy gap as a function of $\phi$. There is one gap closure per cycle, as in Fig. 1(c), taking place at isolated critical values $\phi_{c}$ that are different for the three slabs. An examination of the slab band structures [12] reveals that at $\phi_{c}$ the valence and conduction surface bands touch at a nodal point, which occurs at precisely $E_{\mathrm{F}}$ because we consider the slabs at half filling. If we were to shift $E_{\mathrm{F}}$ away from the nodal point as in Fig. 1(c), each surface would remain metallic over a finite $\phi$ interval containing $\phi_{c}$, as illustrated in Figs. 1(e)-1(g).

The gap closure is pinned to $\phi_{c}=\pi$ on $y_{0}$ surfaces, because at $\phi=\pi$ the system becomes a generalized axion insulator protected by $M_{z}$ and $K C_{2}^{y}$ symmetries, both of which are preserved at those surfaces. ${ }^{2}$ The gap closing occurs exactly at $E=0$ as an artifact of a particle-hole symmetry in the model. As for the $z$-oriented surfaces, neither $M_{z}$ nor $K C_{2}^{y}$ symmetry is preserved there, so these surfaces are not required to be metallic at $\phi=\pi$. Nevertheless they must still become metallic somewhere along the cycle, and the closing of the gap occurs at $\phi_{c}=\pi / 2$ on the $z_{0}$ surface and at $\phi_{c}=3 \pi / 2$ on the $z_{1 / 2}$ surface, again exactly at $E=0$. (The closing occurs at point $\bar{K}$ in the 2D BZ [12], where A and B chains become

\footnotetext{
${ }^{2}$ The symmetry $M_{z}$ (but not $K C_{2}^{y}$ ) is also preserved at $x$-oriented surfaces terminated at armchair edges, and this suffices to pin the gap closure to $\phi_{c}=\pi$ on those surfaces as well. Hence, gapless modes will not appear anywhere along the cycle at hinges adjoining $x$ - and $y$-oriented surfaces.
} 
decoupled, with $H_{\mathrm{A}}=-H_{\mathrm{B}}$. At generic $\phi$ each takes the form of a Rice-Mele chain [18], and the surface gap closure occurs when the surface-state energies of the two chains cross through each other and through zero, which occurs at $\cos \phi=$ 0 where the effective site energy alternation vanishes.)

These gap-closing events at the surfaces are topological phase transitions, and to elucidate the notion of surface topology we now examine the AHC carried by the surfaces along the pumping cycle. For $E_{\mathrm{F}}=0$ and in the limit of a thick slab, we expect the surface AHC to jump by $e^{2} / h$ at $\phi_{c}$, as described by the relation

$$
\sigma_{\mathrm{AHC}}^{\text {surf }}=(n-\theta / 2 \pi) \frac{e^{2}}{h}
$$

between the $\mathrm{AHC}$ of a gapped surface and the bulk axion coupling $[10,20]$. Once a specific branch has been chosen for $\theta$, a unique integer $n$ can be assigned to each surface, and for $n$ to change the surface gap must close and reopen. The difference in AHC between two insulating surface terminations of the same bulk is $\left(e^{2} / h\right) \Delta n$, where $\Delta n$ is the difference between the $n$ values on the two surfaces. In the $\phi$ intervals where $\Delta n$ is nonzero the two surfaces are in topologically distinct states, and if they meet there will be $|\Delta n|$ chiral modes propagating along the adjoining hinge [21].

We have calculated the surface AHC according to Refs. [20,22] for slabs of different thicknesses (7, 13, and 19 cells across $y$, and 7,9, and 11 cells across $z$ ). The extrapolated results are plotted in Fig. 5(b), confirming that Eq. (10) is satisfied throughout the cycle. The AHC of each surface tracks one branch of $-\theta / 2 \pi$ for $0 \leqslant \phi<\phi_{c}$, switches to another branch at $\phi_{c}$, and returns to its initial value at the end of the cycle. We see that the $y_{0}$ surface is topologically distinct from the $z_{0}$ surface for $\phi \in(\pi / 2, \pi)$ and from the $z_{1 / 2}$ surface for $\phi \in(\pi, 3 \pi / 2)$, with $\Delta n=-1$ and +1 , respectively. Gapless modes are therefore expected to appear on the $y_{0} \mid z_{0}$ hinges in the former interval and on the $y_{0} \mid z_{1 / 2}$ hinges in the latter, with opposite chiralities in the two cases. This is illustrated by the outer and middle racetracks in the phase diagram of Fig. 6.

Finally, the topological difference $\Delta n=+1$ between $z_{0}$ and $z_{1 / 2}$ surfaces for $\phi \in(\pi / 2,3 \pi / 2)$ can be understood as follows. To switch from one termination to the other one either removes the outer surface layer or adds an extra layer. Doing so changes the surface $\mathrm{AHC}$ by $\pm e^{2} / h$ in the range $(\pi / 2,3 \pi / 2)$ where the individual layers have Chern numbers \pm 1 , and leaves the surface AHC unchanged in the range $(-\pi / 2, \pi / 2)$ where the layer Chern numbers vanish. A similar behavior was observed in Ref. [22] for a model of an axion insulator, where the half-quantized surface AHC changed sign when a surface layer was added or removed. As a consequence, every single-layer-high surface step carries a chiral edge channel in such cases [23], as shown for our model by the inner racetrack in Fig. 6 .

\section{Gapless hinge modes and hinge Fermi arcs}

To verify the presence of protected hinge modes in the predicted $\phi$ intervals, we have studied rod-shaped samples extended along $x$, and 15 cells thick along both $y$ and $z$. Figure 7(a) shows the energy bands of a $y_{0^{-}}$and $z_{1 / 2^{-}}$ terminated rod at $\phi=5 \pi / 4$ (the middle of the $\phi$ interval

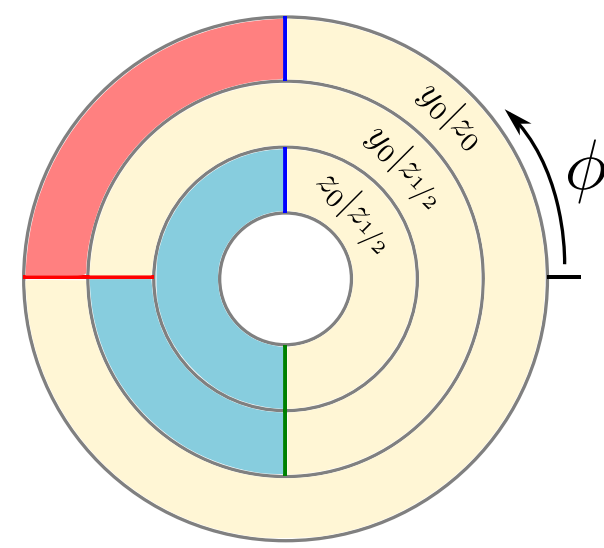

FIG. 6. Topological phase diagram for 1D channels in the alternating Haldane model at half filling. The outer and middle racetracks are for the two types of $y \mid z$ hinges, and the inner one is for singlelayer-high steps on $z$-oriented surfaces. In the yellow regions there are no protected $1 \mathrm{D}$ modes because the surface-AHC difference in Fig. 5 is $\Delta n=0$, while in the blue $(\Delta n=+1)$ and red $(\Delta n=-1)$ regions there is one protected mode per hinge or step. Red, blue, and green lines mark the gap-closing points $\phi_{c}$ on the $y_{0}, z_{0}$, and $z_{1 / 2}$ surfaces, respectively, that separate the different phases.

where gapless hinge modes are expected to occur). All bands are doubly degenerate, since the Kramers-enforcing operator $\Lambda$ of Eq. (9) remains a symmetry of the rod as a whole, and the bands drawn in red and in blue are the predicted hinge modes crossing the bulk gap. The weights of their wave functions on each site are displayed in Fig. 7(b) at an energy near $E=0$ (the middle of the gap); modes localized on adjacent hinges disperse in opposite directions, forming the pattern shown in the inset of panel (a).

The spectrum looks qualitatively the same for any value of $\phi$ between $\pi$ and $3 \pi / 2$; when passing through $\pi$ or $3 \pi / 2$, the surface gap closes and reopens on one of the surfaces, allowing a change of surface topology such that chiral modes are no longer present at the hinges. Outside that interval, the highest-occupied and lowest-unoccupied states become delocalized over the entire rod. When the surface termination is changed from $z_{1 / 2}$ to $z_{0}$ the interval hosting gapless modes changes from $(\pi, 3 \pi / 2)$ to $(\pi / 2, \pi)$ and the chiralities get reversed, as predicted.

Figure 8 shows, for a $y_{0}$ - and $z_{1 / 2}$-terminated rod, the locus of points on the $\left(k_{x}, \phi\right)$ plane where the energy bands cross the Fermi level. In panel (a), the Fermi level is at the charge-neutrality point $E_{\mathrm{F}}=0$. In that case the locus of points at $E_{\mathrm{F}}$ reduces to a fourfold degenerate Fermi arc in $\left(k_{x}, \phi\right)$ space (the same on all hinges). In panel (b) the Fermi level has been shifted to $E_{\mathrm{F}}=0.2$, and as a result the Fermi arc has split into a pair of twofold degenerate arcs, where the Kramers degeneracy again results from the fact that $\Lambda$ of Eq. (9), defined with respect to an inversion center in the middle of the rod, commutes with the rod Hamiltonian. The two Fermi arcs attach tangentially to opposite sides of the two projected surface Fermi surfaces, which have expanded from isolated points in panel (a) to finite disks [compare with Fig. 2(a)]. The way the Fermi arcs close on adjacent hinges is analogous to the way they close on opposite surfaces of a Weyl semimetal slab [15]. 

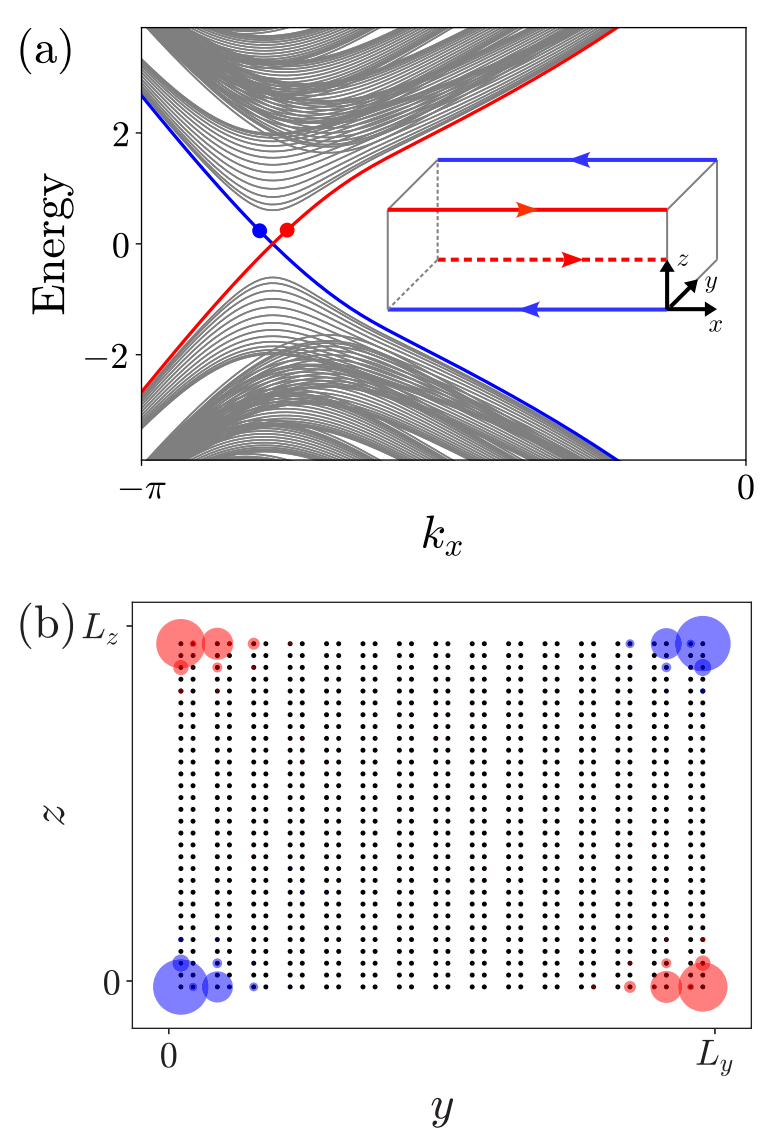

FIG. 7. (a) Energy bands of the alternating Haldane model, calculated at $\phi=5 \pi / 4$ for a rod extended along $x$ and with $y_{0}$ and $z_{1 / 2}$ terminations along $y$ and $z$. All bands are doubly degenerate, and those in red and in blue are hinge-localized chiral modes crossing the bulk and surface gaps, depicted schematically in the inset. (b) Siteresolved weights of the four hinge-localized states at an energy slightly above the crossing point in the middle of the gap, as indicated by the blue and red dots in (a).

\section{Surface-hinge correspondence from slab Wannier bands}

We have seen how the quantized difference in AHC between two surfaces dictates the occurrence of chiral modes on the connecting hinge. In this section we revisit this "surfacehinge correspondence" from the viewpoint of the Wannier band structure of a slab.

\section{Hybrid Wannier representation}

Let us begin by reviewing the hybrid Wannier (HW) representation for a $d$-dimensional insulating crystal [24]. The idea is to describe the valence states using functions that are maximally localized (Wannier-like) along one chosen crystallographic direction $z$, and extended (Bloch-like) along the remaining $d-1$ directions. These HW functions $w_{l n}^{\mathbf{k}}(\mathbf{r})$ are labeled by a wave vector $\mathbf{k}$ in the projected $(d-1)$-dimensional $\mathrm{BZ}$, and by two discrete indices $l$ and $n ; l$ labels cells along $z$, and $n=1, \ldots, J$ is an intracell index with $J$ being the number of valence bands. The HW centers $z_{l n}(\mathbf{k})=\left\langle w_{l n}^{\mathbf{k}}|z| w_{l n}^{\mathbf{k}}\right\rangle$ are organized into "Wannier bands" that are periodic in $z$, with $J$ bands per lattice
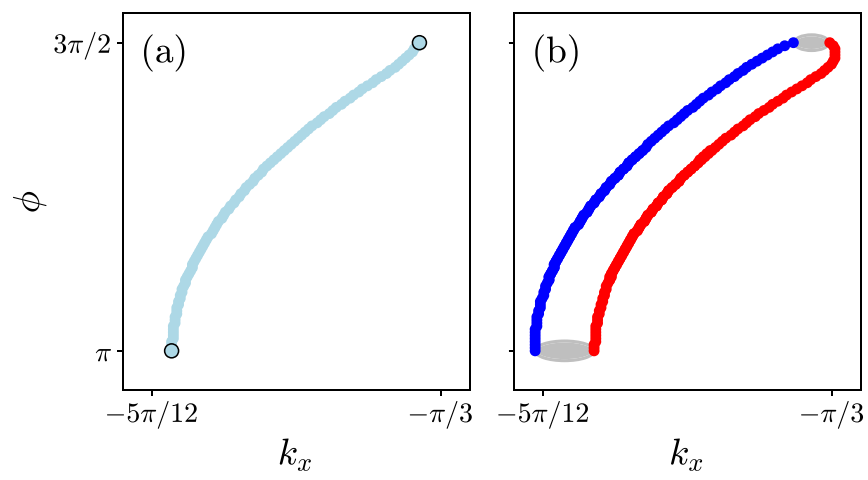

FIG. 8. (a) Fermi arcs traced on the $\left(k_{x}, \phi\right)$ plane by the gapless hinge modes of a rod extended along $x$ and with a $z_{1 / 2}$ vertical termination, for the Fermi level at $E_{\mathrm{F}}=0$. (b) Same, but for $E_{\mathrm{F}}=0.2$. The two elliptical discs indicate approximately the regions where the surface conduction bands move below $E=0.2$.

constant $c$ :

$$
z_{l n}(\mathbf{k})=z_{0 n}(\mathbf{k})+l c .
$$

From now on, the HW centers will be written in units of the lattice constant along the Wannierization direction. Accordingly, we set $c=1$ in Eq. (11).

The Wannier band structure provides a very general means of implementing the bulk-boundary correspondence [25,26]. Consider, for example, a crystal in $d=3$ dimensions. When the boundary of interest is a $z$-terminated surface, one inspects the bulk Wannier bands $z_{\ln }\left(k_{x}, k_{y}\right)$. Under appropriate conditions to be specified shortly, these can be smoothly deformed onto the surface energy bands $E_{n}\left(k_{x}, k_{y}\right)$, so that the topological features of the two spectra are in correspondence: any protected gapless modes in the surface bands are reflected in the connectedness (or "flow") of the Wannier bands [25,26].

For insulators with multiple occupied bands, the ability to make such a smooth deformation depends on the choice of Wannier bands making up a "Wannier unit cell" [12]. This is equivalent to the choice of a Wannier gap separating one Wannier cell from the next along $z$, or, in the language of Ref. [27], "fixing the Wannier chemical potential." Specifically, if the Wannier unit cell is repeated a large integer number of times along $z$, the surface AHC at the top surface of the slab constructed in this way must match that of the insulating surface in question, since if it differs by an integer multiple of the quantum a topological obstruction prevents the smooth deformation. In our case, the correspondence is obvious: setting the Wannier gap at $z=0$ or $1 / 2$ is appropriate for the $z_{0}$ - or $z_{1 / 2}$-terminated surface, respectively. In general, however, a separate calculation may be required to determine the correct choice of Wannier gap for a generic insulating surface.

\section{Flow of surface-localized Wannier bands}

The surface-hinge correspondence can now be developed using closely related methods. To look for protected gapless modes on hinges connecting $y$ - and $z$-oriented surfaces, we examine the Wannier bands $z_{\ln }\left(k_{x}\right)$ of a $y$-terminated slab. The interesting bands are those whose HW functions reside near the surfaces. If the flow of these Wannier bands is such 

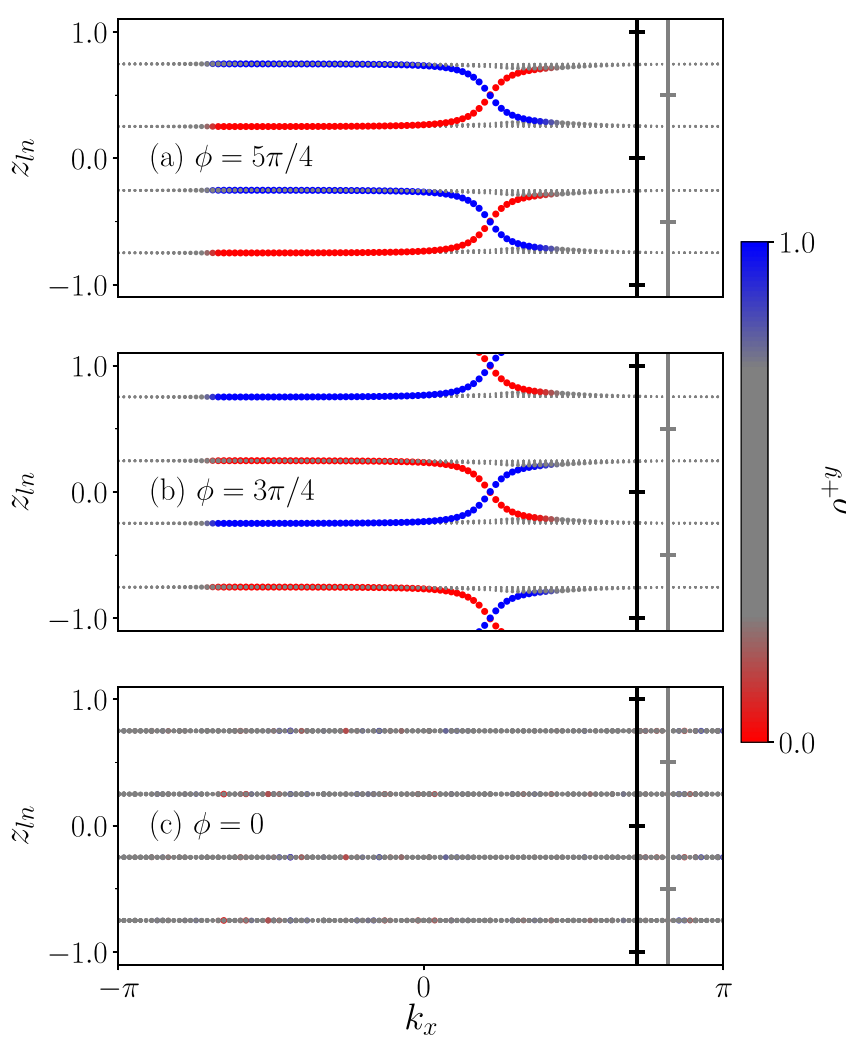

FIG. 9. Wannier bands $z_{l n}\left(k_{x}\right)$ of $y$-terminated slabs of the alternating Haldane model, at different $\phi$ values. The bands are color coded according to the degree of localization on the $+\hat{\mathbf{y}}$ surface [Eq. (12)]: gray dots are modes extending along $y$ across the entire slab, and blue (red) dots are modes localized on the $+\hat{\mathbf{y}}(-\hat{\mathbf{y}})$ surface; the degree of surface localization is also indicated by the size of the dots. Two types of cells are displayed in each panel: the " $z_{0}$ cell" with boundaries at $z=0 \bmod 1$ (in black), and the " $z_{1 / 2}$ cell" with boundaries at $z=1 / 2 \bmod 1$ (in gray).

as to cross the Wannier gap appropriate to the $z$-terminated surface of interest, then the $x$-directed $y \mid z$ hinges will host topologically protected gapless modes. Identical conclusions are reached by examining the bands $y_{l n}\left(k_{x}\right)$ of $z$-terminated slabs.

Figure 9 shows the bands $z_{\ln }\left(k_{x}\right)$ of $y_{0}$-terminated slabs with a thickness of 20 unit cells, calculated at $\phi=5 \pi / 4$, $3 \pi / 4$, and zero. They are color coded by the weight

$$
\rho_{n}^{+y}\left(k_{x}\right)=\int_{+y}\left|w_{l n}^{k_{x}}(\mathbf{r})\right|^{2} d^{3} r
$$

of the HW functions in the half of the slab containing the $+\hat{\mathbf{y}}$ surface, and for added clarity the degree of localization at the surfaces is also indicated by the size of the dots.

Let us first examine the bands at $\phi=5 \pi / 4$ in panel (a). At $k_{x}=-\pi$ they are evenly split into two narrow bulklike groups, one centered at $z=1 / 4 \bmod 1$ and another at $z=$ $3 / 4 \bmod 1$. Between them there is a " $z_{0}$ gap" centered at $z=0 \bmod 1$, and a " $z_{1 / 2}$ gap" centered at $z=1 / 2 \bmod 1$. As $k_{x}$ increases the two groups broaden slightly, and one band detaches from each. The two detached bands cross the $z_{1 / 2}$ gap in opposite directions, and as $k_{x}$ approaches $\pi$ each merges with the bulklike group from which the other came. While

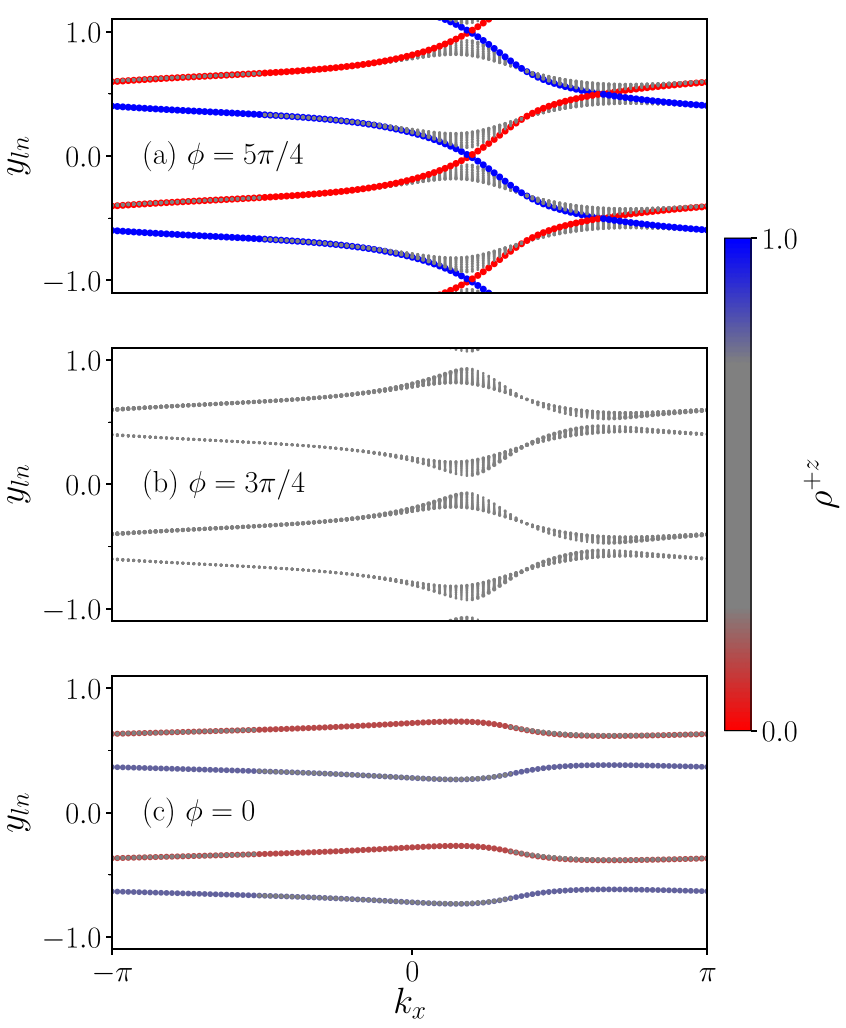

FIG. 10. Same as Fig. 9, but for the Wannier bands $y_{l n}\left(k_{x}\right)$ of $z_{1 / 2}$-terminated slabs.

crossing the gap, these two chiral bands become strongly localized on opposite surfaces; this surface-localized flow across the $z_{1 / 2}$ gap is maintained over the interval $\pi<\phi<$ $3 \pi / 2$, signaling the presence of protected gapless modes on $y_{0} \mid z_{1 / 2}$ hinges. ${ }^{3}$ Conversely, the lack of flow on the $z_{0}$ gap indicates the absence of such modes on $y_{0} \mid z_{0}$ hinges over that interval.

The same logic applies to the other panels of Fig. 9. In panel (b) the Wannier flow at $\phi=3 \pi / 4$ shifts to the $z_{0}$ gap (and switches chirality), consistent with the fact that the $z_{0}$ termination is the one producing hinge modes (of the opposite chirality), for $\pi / 2<\phi<\pi$. In panel (c) both types of gaps are devoid of chiral surface modes at $\phi=0$, reflecting the absence of chiral hinge modes for $-\pi / 2<\phi<\pi / 2$ with either type of $z$ termination.

With the above procedure, we have been able to predict the existence of gapless modes on both $y_{0} \mid z_{0}$ and $y_{0} \mid z_{1 / 2}$ hinges from a single slab calculation (at each $\phi$ ). This is somewhat unexpected, given that the surface-AHC approach of Sec. III B required three separate slab calculations to gather the same information. It should be noted, however, that the HW-based procedure only works when the choice of Wannier gap corresponding to the $z$-terminated surface of interest is known, whereas the surface-AHC approach can be applied directly to arbitrary insulating surfaces.

${ }^{3}$ In Fig. 9(a), the Wannier band localized on the $+\hat{\mathbf{y}}$ surface flows downward, in agreement with the negative chirality of the mode localized at the hinge between the $+\hat{\mathbf{y}}$ and $+\hat{\mathbf{z}}$ surfaces in Fig. 7. 

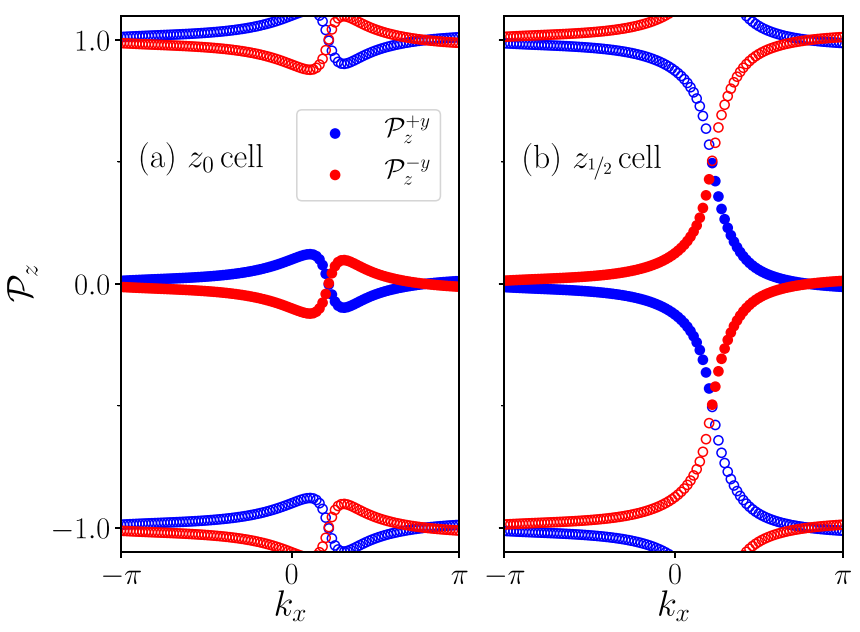

FIG. 11. Pumped charge $\mathcal{P}_{z}\left(k_{x}\right)$ of Eq. (13), in units of $e$, for a $y$-terminated slab at $\phi=5 \pi / 4$. Blue (red) curves denote results for the $+\hat{\mathbf{y}}(-\hat{\mathbf{y}})$ surface. In (a) and (b), Eq. (13) is evaluated using the $z_{0}$ and $z_{1 / 2}$ cells shown in Fig. 9, respectively. Filled dots correspond to the home cell $[[0,1]$ in (a) and $[-1 / 2,1 / 2]$ in (b)], and open dots correspond to the cells immediately above and below.

The protected modes on $y \mid z$ hinges can also be deduced from the Wannier spectrum $y_{n}\left(k_{x}\right)$ of $z$-terminated slabs, but this requires two slab calculations instead of one (one for each type of $z$ termination). This is illustrated in Fig. 10 for the case of $y_{0} \mid z_{1 / 2}$ hinges, using $z_{1 / 2}$-terminated slabs. As expected, Wannier flow is present on the $y_{0}$ gap at $\phi=5 \pi / 4$ but not at $\phi=3 \pi / 4$ or 0 .

\section{Interpretation in terms of charge pumping at the surface}

The information in Figs. 9 and 10 about the topology of $y \mid z$ hinges can be presented concisely in the language of charge pumping. Let us describe the procedure for the case of Fig. 9, where the slab is terminated along $y$. Following Ref. [28], we assign to the $+\hat{\mathbf{y}}$ surface a quantity with units of charge defined as

$$
\mathcal{P}_{z}^{+y}\left(k_{x}\right)=-\frac{e}{c} \sum_{n} z_{0 n}\left(k_{x}\right) \rho_{n}^{+y}\left(k_{x}\right),
$$

where $\rho_{n}^{+y}$ is given by Eq. (12). For a given value of $k_{x}$, this quantity is a measure of the charge pumped along $z$ on the $+\hat{\mathbf{y}}$ edge of the ribbon (finite in $y$, infinite in $z$ ) described by $H_{\text {slab }}\left(k_{x}\right)$. However, its physical interpretation is rather subtle. For example, consider a weak electric field along $x$ that acts for one Bloch period. In this case, the continuous change in $\mathcal{P}_{z}^{+y}$ as $k_{x}$ increases by $2 \pi$ is quantized in units of $e$, and it describes the $+\hat{\mathbf{z}}$-directed flow of current on the $+\hat{\mathbf{y}}$ surface relative to the $-\hat{\mathbf{y}}$-directed current on the $+\hat{\mathbf{z}}$ surface (that is, the quantized difference $\Delta n$ in surface AHC). Note that Eq. (13) depends on the choice of Wannier unit cell, and again the answer will only be correct if that cell is chosen correctly for the $+\hat{\mathbf{z}}$-terminated surface of interest.

In Fig. $11, \mathcal{P}_{z}^{+y}$ is plotted at $\phi=5 \pi / 4$ for two different cell choices. In panel (a), the black $z_{0}$ cell in Fig. 9 was used. Since in Fig. 9(a) the chiral Wannier band localized on the $+\hat{\mathbf{y}}$ surface does not cross the boundaries of that cell, $\mathcal{P}_{z}^{+y}$ does not exhibit flow as a function of $k_{x}$, indicating that no protected gapless modes are present on the $y_{0} \mid z_{0}$ hinges. In Fig. 11(b) the calculation was repeated using the gray $z_{1 / 2}$ cell in Fig. 9. Now the surface-localized band does cross the cell boundaries, and as a result $\mathcal{P}_{z}^{+y}$ exhibits flow as a function of $k_{x}$ (when viewed as a continuous but multivalued function), indicating the presence of gapless modes on the $y_{0} \mid z_{1 / 2}$ hinges. Also shown in Fig. 11 is $\mathcal{P}_{z}^{-y}$, obtained by replacing $\rho_{n}^{+y}$ with $\rho_{n}^{-y}=1-\rho_{n}^{+y}$ in Eq. (13).

\section{SUMMARY AND OUTLOOK}

We have shown that gapless modes appear naturally on the hinges of $3 \mathrm{D}$ insulators undergoing an axion pumping cycle. The basic idea is illustrated in Figs. 1 and 2. When a surface is introduced in the system, the valence and conduction surface bands must exhibit at least one nodal touching along the cycle. If, as is generically the case, those band touchings occur on adjacent surfaces at different values of the pumping parameter $\phi$, then the connecting hinge will host chiral modes over the intervening $\phi$ range. Those modes are boundary manifestations of the second-Chern number characterizing the axion pump, and they can be viewed as Fermi arcs in the BZ of the 2D hinge connecting the 3D surfaces of a $4 \mathrm{D}$ sample with $\left(k_{x}, k_{y}, k_{z}, \phi\right)$ reciprocal space.

Note that at any given value of $\phi$ the appearance of 1D modes on the hinges of the 3D crystal represents an "extrinsic" higher-order topological phase in the language of Refs. [14,29], since the bulk is topologically trivial and hinge modes are not required. Instead, the presence of Fermi arc states is generically required on the $2 \mathrm{D}$ surfaces of the $4 \mathrm{D}$ second-Chern insulator, thus representing "intrinsic" topology when the system is viewed from the standpoint of the global $\left(k_{x}, k_{y}, k_{z}, \phi\right)$ parameter space.

We have exemplified these behaviors by means of a tightbinding model, but the same methodology could easily be applied in the framework of ab initio calculations. However, it remains a major challenge to devise a physical mechanism leading to the adiabatic pumping of axion coupling in a real material.

Alternatively, it may be possible to demonstrate axion pumping behavior in other settings such as photonic crystals, ultracold atoms, or electrical circuits. The physics of secondChern insulators is already being explored in such systems [30-37], and we hope that the present paper may inspire future efforts towards the observation of the associated topological hinge states.

\section{ACKNOWLEDGMENTS}

Work by T.O. was funded by the Danish Independent Research Foundation Grant No. 6108-00464B. Work by T.R. was supported by the Forschungsstipendium Grant No. RA 3025/1-1 from the Deutsche Forschungsgemeinschaft. Work by D.V. was supported by National Science Foundation Grant No. DMR-1954856. Work by I.S. was supported by Grant No. FIS2016-77188-P from the Ministerio de Ciencia e Innovación (Spain). 
[1] D. Vanderbilt, Berry Phases in Electronic Structure Theory (Cambridge University, Cambridge, England, 2018).

[2] J. Zak, Berry's Phase for Energy Bands in Solids, Phys. Rev. Lett. 62, 2747 (1989).

[3] R. D. King-Smith and D. Vanderbilt, Theory of polarization of crystalline solids, Phys. Rev. B 47, 1651 (1993).

[4] D. Vanderbilt and R. D. King-Smith, Electric polarization as a bulk quantity and its relation to surface charge, Phys. Rev. B 48, 4442 (1993).

[5] D. J. Thouless, Quantization of particle transport, Phys. Rev. B 27, 6083 (1983).

[6] F. D. M. Haldane, Model for a Quantum Hall Effect without Landau Levels: Condensed-Matter Realization of the "Parity Anomaly", Phys. Rev. Lett. 61, 2015 (1988).

[7] C.-Z. Chang, J. Zhang, X. Feng, J. Shen, Z. Zhang, M. Guo, K. Li, Y. Ou, P. Wei, L.-L. Wang, Z.-Q. Ji, Y. Feng, S. Ji, X. Chen, J. Jia, X. Dai, Z. Fang, S.-C. Zhang, K. He, Y. Wang, L. Lu, X.-C. Ma, and Q.-K. Xue, Experimental observation of the quantum anomalous Hall effect in a magnetic topological insulator, Science 340, 167 (2013).

[8] C.-Z. Chang, W. Zhao, D. Y. Kim, H. Zhang, B. A. Assaf, D. Heiman, S.-C. Zhang, C. Liu, M. H. W. Chan, and J. S. Moodera, High-precision realization of robust quantum anomalous Hall state in a hard ferromagnetic topological insulator, Nat. Mater. 14, 473 (2015).

[9] X.-L. Qi, T. L. Hughes, and S.-C. Zhang, Topological field theory of time-reversal invariant insulators, Phys. Rev. B 78, 195424 (2008).

[10] A. M. Essin, J. E. Moore, and D. Vanderbilt, Magnetoelectric Polarizability and Axion Electrodynamics in Crystalline Insulators, Phys. Rev. Lett. 102, 146805 (2009).

[11] M. Taherinejad and D. Vanderbilt, Adiabatic Pumping of Chern-Simons Axion Coupling, Phys. Rev. Lett. 114, 096401 (2015).

[12] T. Olsen, M. Taherinejad, D. Vanderbilt, and I. Souza, Surface theorem for the Chern-Simons axion coupling, Phys. Rev. B 95, 075137 (2017).

[13] F. Schindler, A. M. Cook, M. G. Vergniory, Z. Wang, S. S. P. Parkin, B. A. Bernevig, and T. Neupert, Higher-order topological insulators, Sci. Adv. 4, eaat0346 (2018).

[14] M. Geier, L. Trifunovic, M. Hoskam, and P. W. Brouwer, Second-order topological insulators and superconductors with an order-two crystalline symmetry, Phys. Rev. B 97, 205135 (2018).

[15] N. P. Armitage, E. J. Mele, and A. Vishwanath, Weyl and Dirac semimetals in three-dimensional solids, Rev. Mod. Phys. 90, 015001 (2018).

[16] H. B. Nielsen and M. Ninomiya, The Adler-Bell-Jackiw anomaly and Weyl fermions in a crystal, Phys. Lett. B 130, 389 (1983).

[17] F. D. M. Haldane, Attachment of surface "Fermi arcs" to the bulk Fermi surface: "Fermi-level plumbing" in topological metals, arXiv:1401.0529.

[18] M. J. Rice and E. J. Mele, Elementary Excitations of a Linearly Conjugated Diatomic Polymer, Phys. Rev. Lett. 49, 1455 (1982)

[19] N. Varnava, I. Souza, and D. Vanderbilt, Axion coupling in the hybrid Wannier representation, Phys. Rev. B 101, 155130 (2020).
[20] T. Rauch, T. Olsen, D. Vanderbilt, and I. Souza, Geometric and nongeometric contributions to the surface anomalous Hall conductivity, Phys. Rev. B 98, 115108 (2018).

[21] M. Sitte, A. Rosch, E. Altman, and L. Fritz, Topological Insulators in Magnetic Fields: Quantum Hall Effect and Edge Channels with a Nonquantized $\theta$ Term, Phys. Rev. Lett. 108, 126807 (2012).

[22] N. Varnava and D. Vanderbilt, Surfaces of axion insulators, Phys. Rev. B 98, 245117 (2018).

[23] R. S. K. Mong, A. M. Essin, and J. E. Moore, Antiferromagnetic topological insulators, Phys. Rev. B 81, 245209 (2010).

[24] D. Gresch, G. Autès, O. V. Yazyev, M. Troyer, D. Vanderbilt, B. A. Bernevig, and A. A. Soluyanov, Z2Pack: Numerical implementation of hybrid Wannier centers for identifying topological materials, Phys. Rev. B 95, 075146 (2017).

[25] L. Fidkowski, T. S. Jackson, and I. Klich, Model Characterization of Gapless Edge Modes of Topological Insulators Using Intermediate Brillouin-Zone Functions, Phys. Rev. Lett. 107, 036601 (2011).

[26] T. Neupert and F. Schindler, Lecture notes on topological crystalline insulators, in Topological Matter-Lectures from the Topological Matter School 2017, edited by D. Bercioux, J. Cayssol, M. G. Vergniory, and M. Reyes Calvo (Springer, New York, 2018), Chap. 2, p. 31.

[27] E. Khalaf, W. A. Benalcazar, T. L. Hughes, and R. Queiroz, Boundary-obstructed topological phases, arXiv:1908.00011.

[28] W. A. Benalcazar, B. A. Bernevig, and T. L. Hughes, Electric multipole moments, topological multipole moment pumping, and chiral hinge states in crystalline insulators, Phys. Rev. B 96, 245115 (2017).

[29] L. Trifunovic and P. W. Brouwer, Higher-Order Bulk-Boundary Correspondence for Topological Crystalline Phases, Phys. Rev. X 9, 011012 (2019).

[30] T. Ozawa, H. M. Price, N. Goldman, O. Zilberberg, and I. Carusotto, Synthetic dimensions in integrated photonics: From optical isolation to four-dimensional quantum Hall physics, Phys. Rev. A 93, 043827 (2016).

[31] O. Zilberberg, S. Huang, J. Guglielmon, M. Wang, K. P. Chen, Y. E. Kraus, and M. C. Rechtsman, Photonic topological boundary pumping as a probe of 4D quantum Hall physics, Nature (London) 553, 59 (2018).

[32] L. Lu, H. Gao, and Z. Wang, Topological one-way fiber of second Chern number, Nat. Commun. 9, 5384 (2018).

[33] X. Zhang, Y. Chen, Y. Wang, Y. Liu, J. Y. Lin, N. C. Hu, B. Guan, and C. H. Lee, Entangled four-dimensional multicomponent topological states from photonic crystal defects, Phys. Rev. B 100, 041110(R) (2019).

[34] H. M. Price, O. Zilberberg, T. Ozawa, I. Carusotto, and N. Goldman, Four-Dimensional Quantum Hall Effect with Ultracold Atoms, Phys. Rev. Lett. 115, 195303 (2015).

[35] H. M. Price, O. Zilberberg, T. Ozawa, I. Carusotto, and N. Goldman, Measurement of Chern numbers through center-of-mass responses, Phys. Rev. B 93, 245113 (2016). 
[36] M. Lohse, C. Schweizer, H. M. Price, O. Zilberberg, and I. Bloch, Exploring 4D quantum Hall physics with a 2D topological charge pump, Nature (London) 553, 55 (2018).
[37] M. Ezawa, Electric circuit simulations of $n$ th-Chern-number insulators in $2 n$-dimensional space and their non-Hermitian generalizations for arbitrary $n$, Phys. Rev. B 100, 075423 (2019). 